\title{
A COMPARISON OF FOOD PORTION SIZE ESTIMATION BY OLDER ADULTS, YOUNG ADULTS AND NUTRITIONISTS
}

\author{
C.M. TIMON ${ }^{1}$, S.E. COOPER ${ }^{2}$, M.E. BARKER ${ }^{2}$, A.J. ASTELL ${ }^{3}$, T. ADLAM ${ }^{4}$, \\ F. HWANG ${ }^{5}$, E.A. WILLIAMS ${ }^{2}$
}

1. Institute of Food and Health, University College Dublin, Belfield, Dublin 4, Ireland; 2. Human Nutrition Unit, Department of Oncology \& Metabolism, University of Sheffield, Sheffield S10 2RX, United Kingdom; 3. School of Health and Related Research, Faculty of Medicine, Dentistry \& Health, University of Sheffield, Sheffield, S1 4DA, United Kingdom and Ontario Shores Centre for Mental Health Sciences, Whitby, Ontario, L1N 559, Canada; 4. BIME, Royal United Hospital, Bath, BA1 3NG, United Kingdom; 5. School of Systems

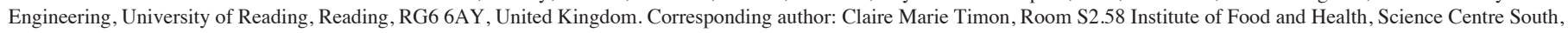
University College Dublin, Belfield, Dublin 4, Ireland, Email: claire.timon@ucd.ie, Phone: 0035317162442, Fax: 0035317166104

\begin{abstract}
Objectives: To investigate the ability of older adults, younger adults and nutritionists to assess portion size using traditional methods versus a computer-based method. This was to inform the development of a novel dietary assessment method for older adults "The NANA system". Design: Older and younger adults assessed the portion size of self-served portions of foods from a buffet style set up using traditional and computerised portion size assessment aids. Nutritionists assessed the portion size of foods from digital photographs using computerised portion size aids. These estimates were compared to known weights of foods using univariate analyses of covariance (ANCOVA). Setting: The University of Sheffield, United Kingdom. Subjects: Forty older adults (aged 65 years and over), 41 younger adults (aged between 18 and 40 years) and 25 nutritionists. Results: There was little difference in the abilities of older and younger adults to assess portion size using both assessment aids with the exception of small pieces morphology. Even though the methods were not directly comparable among the test groups, there was less variability in portion size estimates made by the nutritionists. Conclusion: Older adults and younger adults are similar in their ability to assess food portion size and demonstrate wide variability of estimation compared to the ability of nutritionists to estimate portion size from photographs. The results suggest that the use of photographs of meals consumed for portion size assessment by a nutritionist may improve the accuracy of dietary assessment. Improved portion size assessment aids are required for all age groups.
\end{abstract}

Key words: Portion size assessment, older adults, young adults, nutritionists .

\section{Introduction}

Portion size estimation has been recognised as one of the main sources of inaccuracy in nutritional assessment (1). Accurate dietary intake information is essential to properly evaluate an individual's diet relative to recommendations, to relate dietary intakes to health and disease and lastly evaluate the effectiveness of interventions (2). Most dietary assessment methods require a food portion size to be identified or assigned to each food/drink consumed in order to calculate the corresponding nutrient value of that food item. In the past, food records with weighed intakes were considered the gold standard method of dietary assessment, but for many populations it has been shown that recording intake using this method has resulted in misreporting of dietary intake potentially due to the participant burden associated with this method (3). It has been shown that estimated food records provided improved participation rates and data quality in comparison to more burdensome methods such as weighed food records (4). Estimated food records are typically achieved by interviewing the participant after a period of dietary recording in order to gather information about the portion size of foods consumed, an approach that utilises portion size assessment aids such as food models or a food photograph atlas (5). Several studies have explored portion size assessment; however few have investigated older adults' assessment of portion size using traditional methodologies. There are exceptions whereby investigators concluded that older adults were less accurate in their ability to assess portion size when compared with other age groups (5-7). Previous studies investigating the ability of children to estimate portion size suggest that immature cognitive skills may prevent them from accurately assessing portion size even when visual aids are used (8). Impaired cognitive function and reduced attention span associated with ageing (9) may, in part, explain older adults' reduced capacity to accurately assess portion size. Impaired eyesight in older age may also contribute to the inaccuracies in portion size assessment encountered in these studies.

Attempts have been made to reduce the errors associated with estimation of portion sizes using computer based technology $(10,11)$. The Interactive Portion Size Assessment System (IPSAS) tool (10) is a computerised portion size assessment aid designed specifically for children using agespecific portion size photographs. The IPSAS tool was designed to assist portion size estimation during dietary assessment with children and displays digital portion size photographs and depicts photographs of leftover portions also. Compared with traditional portion size assessment aids IPSAS has been shown to provide acceptable estimates of portion size demonstrating the feasibility of utilising computer based technology for portion size assessment in children. The ability of adults to estimate portion size using digital portion size images was 


\section{THE JOURNAL OF NUTRITION, HEALTH \& AGING@}

investigated (11). Forty-nine adult participants assessed the portion size of self-served food portions using a computerised software program which displayed digital portion size images as assessment aids. Different sets of images were used as assessment aids including: images with different sizes (large vs. small), images from different angles (aerial, angled) and different quantities of images displayed at a time (4 vs. 8). Although results were not statistically significant, Subar concluded that the use of aerial photographs and the use of 8 vs. 4 images for portion size assessment yielded the most accurate estimations.

The use of computerised image analysis to determine the portion size of food from photographs of meals has also been investigated (12-14). Image analysis offers the potential of automating food recognition alongside volume estimation, which could improve the accuracy of portion size assessment and remove the burden of portion size assessment from the participant. The majority of these analysis techniques are still being developed and may take some time before they can be applied to dietary assessment as challenges such as the positioning of food on a plate and the differentiation of foods of a similar colour have proven difficult to overcome. Wang et al. (15) and Rollo et al. (16) investigated the feasibility of the use of Personal Digital Assistant (PDA) and mobile phone devices respectively to remove the onus of portion size assessment necessary for dietary assessment from the participant. The devices were used by adult participants to take photographs of food and drink items and were sent to dieticians alongside descriptions of what was consumed for retrospective portion size and subsequent nutrient intake analysis. Rollo et al. (16) noted that the use of voice recordings to describe what was consumed was often insufficient for the coding of data whereas Wang et al. (15) observed good agreement when descriptions of items consumed were provided by participants (using a stylus to write on the photographs captured by the PDA) alongside photographs of meals compared to a 1 day food diary.

The overall aim of this study was to investigate the ability of older adults, younger adults and nutritionists to accurately assess portion size using traditional versus technology-based portion size assessment aids. The results of this project were used to inform the development of the dietary component of a computer based assessment tool for older adults, i.e. the "NANA" (Novel Assessment of Nutrition and Ageing) system $(17,18)$. In terms of the portion size assessment component of the NANA system, two possible strategies for assessing portion size were considered. The first was asking older adults to assess the portion size of meals themselves using either computerised or traditional portion size aids. The second was the possibility of participants photographing all food consumed for subsequent assessment by a nutritionist. It was therefore necessary to explore the effect of age and methodology on the accuracy of portion size assessment and also explore the ability of nutritionists to assess portion size from photographs of foods.

\section{Methods}

\section{Participants}

Older adults, aged 65 years and over and younger adults, aged between 18 and 40 years, were recruited in Sheffield, UK. Older adults were recruited from the general public via older adult community groups and advertisement in local newsletters distributed in different areas of Sheffield in an attempt to recruit participants that were representative of the general older adult population. Younger adults were recruited from the staff and student population of The University of Sheffield and the early career nutritionists (masters students) were recruited from the University of Sheffield's MMedSci course in Human Nutrition. All the nutritionists were in the second semester of the masters course and had received training on how to use portion size assessment aids via workshops during the first semester and experienced using the portion size aids in dietary assessment practicals throughout the year. For the computerised portion size assessment aid the researcher controlled the software for all participants. This study was approved by the University of Sheffield's Ethics Committee (SMBRER140) and all participants provided written, informed consent prior to the study.

\section{Portion size assessment aids}

The Food Photographic Atlas of Food Portion Sizes (19) provided the basis for the two portion size assessment aids used in this study. The atlas consists of a series of photographs for 78 foods. For the majority of food types (excluding spreads) there is a series of 8 photographs depicting a different portion size from the 5th and 95th centile (representing the lowest and the highest portion size) of the distribution of portion sizes consumed by adults in the UK (20). The atlas was used in 2 formats. First was the traditional paper format, where participants were asked to select the most representative photograph from a hardcopy of a portion size book. The second format utilised a computerised version of the food portion size photographs using software developed at the University of Reading. In this format, portion size images were displayed on the computer screen two at a time. A researcher controlled the software and participants instructed the researcher to move through the pictures at a speed that was convenient to them. The participant then told the researcher which picture they felt most closely represented the portion of food in front of them. This helped to assess the feasibility of embedding this style of portion size assessment into software for older adults.

\section{Study design and procedure}

Participants were invited to attend the Clinical Research Facility, Royal Hallamshire Hospital, Sheffield, UK. Participants were asked to complete a baseline demographic questionnaire which also included items about information which could possibly affect an individuals' ability to estimate portion size using traditional and computerised methods (e.g. 
PORTION ESTIMATION BY OLDER AND YOUNG ADULTS

Table 1

Food morphology and meals served by participants

\begin{tabular}{lllll}
\hline Meal number & Amorphous & Large pieces & Small pieces & Spreads \\
\hline Meal 1 & Chicken curry & Potatoes & Peas & Butter on Bread \\
Meal 2 & Irish stew & Potatoes & Mixed vegetables & Butter on cracker \\
Meal 3 & & Cucumber slices & Grated cheese & Quiche, Sponge cake \\
Meal 4 & & Tomato slices & Grated carrot & Meat pie, Cheesecake \\
\hline
\end{tabular}

medical conditions, eyesight and previous experience with technology).

\section{Data Collection}

\section{Younger and Older adults}

Each participant was individually presented with a food buffet containing 14 food items (spreads and potatoes were assessed twice). Food items were included in the buffet on the basis of their food form and whether portion size photographs were available for these foods. The food categories included were based on those used in similar studies $(10,11)$. The 14 different foods represented five food morphology categories (amorphous, small pieces, large pieces, shaped and spread) (Table 1). These foods were grouped into 4 different combinations of morphologies (meals) and for each meal; participants were provided with a clean plate and instructed to serve portions (of foods that made up each meal combination under instruction of the researcher) that they would normally serve for themselves or somebody else. All participants served the meal combinations in the same order. The portion size assessment of spreads and potatoes was duplicated to investigate if this differed depending on other meal constituents. The serving bowls in which the buffet items were presented in were weighed before and after the participant had served a portion of food to determine the actual weight of food that had been served.

Participants were then asked to assess the portion size of the food they served themselves with the served plate of food in front of them. The purpose of this was to replicate what a user may be asked to do in their own homes if being asked to assess portion size as part of a dietary food record. Each participant assessed two meals using the traditional food atlas and two meals using the computer format in random order i.e. all participants used both portion size assessment aids but in a different order.

\section{Nutritionists}

We wished to explore whether food images captured by participants could be used by nutritionists to estimate food portion size, and so in contrast to the older and younger adults, the nutritionists were asked to estimate food portion sizes from digital photographs of non-self-served meals. The nutritionists were shown images of 4 meals which were photographed by researchers. Photographs of the meals were displayed on a computer screen and the nutritionists were instructed to identify the portion sizes of food served using only the computerised version of the food atlas.

\section{Data analysis}

All statistical analysis was performed in SPSS (Version 19, Illinois USA). Results are described at a group level with means and standard deviations. The differences between the mean gram weights of individual food items actually served and mean gram weights of individual food items estimated using the photographs was calculated. To identify if the participant had selected the correct portion size photograph for the portion they had actually served, ranges of weight were assigned to each of the 8 portion size photographs for each food by calculating the weight difference between the 8 portion photographs. The portion size photographs increased in weight incrementally between each picture, but this value differed depending on the food type. The actual corresponding gram weight of the portion depicted in the photograph was used in the analysis. The correct portion size photograph was identified as the one corresponding most closely to the weight of the food item served. Underestimation was defined by the selection of any portion size photographs lower than the correct photograph, and overestimation was denoted by the selection of any portion size photographs greater than the correct photograph.

Analysis was performed by calculating the ratio of the gram weight of the food atlas photograph the participant selected (estimate) to the gram weight of the correct food atlas photograph (actual). A value greater than 1 indicated over reporting and a value less than 1 indicated underreporting. Univariate analyses of covariance (ANCOVA) were used to identify the effect of age and method on portion size assessment of 5 different food morphologies. For analysis investigating the effect of participant age on portion size assessment, the method (computerised or traditional paper portion size atlas) and individual food items within the respective food morphology category were used as covariates in the analysis. Similarly for analysis investigating the effective of method on portion size assessment, the individual food items which make up the respective food morphology and participant age were used as covariates. 
THE JOURNAL OF NUTRITION, HEALTH \& AGINGC

Table 2

Demographic characteristics of participants

\begin{tabular}{llll}
\hline Study demographics $(\mathbf{n}=\mathbf{1 0 6})$ & Older Adults $(\mathbf{n}=\mathbf{4 0})$ & Young Adults $(\mathbf{n}=\mathbf{4 1})$ & Nutritionists $(\mathbf{n}=\mathbf{2 5})$ \\
\hline Age $($ mean, SD*) & 73 years $( \pm 5.8)$ & 29 years $( \pm 6.74)$ & 25 years $( \pm 2.68)$ \\
Gender & & & \\
$\%$ Males & 37.5 & 22 & 33 \\
$\quad \%$ Females & 62.5 & 78 & 67 \\
Participants with $\geq 1$ medical condition & $72.5 \%$ & $7.30 \%$ & $11 \%$ \\
Can read newspaper with/without glasses (\% Yes) & $97.5 \%$ & $100 \%$ & $100 \%$ \\
No difficulty preparing food (\% Yes) & $90 \%$ & $100 \%$ & $100 \%$ \\
Arthritis (\% Yes) & $22.5 \%$ & $0 \%$ & $0 \%$ \\
Hearing problems (\% Yes) & $32.5 \%$ & $9.80 \%$ & $0 \%$ \\
Technology usage (\% Yes) & & & \\
Have you previously used a mobile phone? & $90 \%$ & $100 \%$ & $100 \%$ \\
Have you previously used a digital camera? & $62.5 \%$ & $100 \%$ & $100 \%$ \\
Have you previously used a computer? & $77.5 \%$ & $100 \%$ & $100 \%$ \\
Have you previously used self-service check outs? & $62.5 \%$ & $95.1 \%$ & $100 \%$ \\
Do you have internet at home? & $75 \%$ & $100 \%$ & $100 \%$ \\
Do you use the internet? & $72.5 \%$ & $100 \%$ & $100 \%$ \\
\hline
\end{tabular}

*SD, standard deviation

Due to the fact that the nutritionists estimated portion sizes from photographs, the results of this group are not directly comparable to the younger and older adults. However the overall mean ratio of estimated vs. actual weight was calculated for each test population and were used alongside the minimum and maximum ratio range to compare all three groups' ability to estimate portion size. Univariate analysis of covariance (ANCOVA) was used to identify the difference in ability to estimate portion size between the 3 groups.

\section{Results}

One hundred and six participants took part in the study (40 older adults, 41 young adults and 25 nutritionists). The demographic characteristics and technology usage reported by study participants are shown in Table 2. Despite efforts made to recruit older adult participants that were representative of the general older adult population, the majority of participants recruited were actively involved in community groups and were motivated to be involved in research. Participants of all groups had high functioning eye sight except one older adult who brought a magnifying glass to assist portion size assessment. Ninety percent of older participants had previously used a mobile phone and over $72 \%$ of the older participants used the internet on a regular basis. All younger adults and nutritionists used all the technology specified in the questionnaire (mobile phone, digital camera, computer, microwave etc.) with the exception of one younger adult who had not previously used a self-service checkout. The results of the ANCOVA show there was no interaction $(\mathrm{F}=0.029, \mathrm{df}=1, \mathrm{P}=0.864)$ between method (traditional atlas vs. computerised atlas) and age (older vs. younger adults) in the assessment of portion size. The data from younger and older adults was combined to examine the effect of method on the portion size assessment of different food morphologies and similarly the data for both methods was combined to further examine the effect of age on the portion size assessment on different food morphologies.

\section{Older adults versus young adults}

The ratio of estimated portion size weights to actual portion size weights for foods assessed by older and younger adults is shown in Figure 1. For this analysis foods have been categorised according to their morphology. There was a significant difference between the ability of older and younger adults in the assessment of "small pieces" foods which represent food items such as peas and grated cheese $(\mathrm{F}=4.66, \mathrm{df}=1, \mathrm{P}=0.032$ ). Older adults' overestimated spreads compared to younger adults however this overestimation was not significant. Both older and younger adults overestimated amorphous foods and underestimated large pieces but were very accurate in the portion size assessment of shaped foods. Over and underestimation by older and younger adults was in the same direction for each of the food types suggesting that the perception of portion size is similar for both older and young adults. 
Figure 1

The ability of older and younger adults to assess food portion size according to food morphology

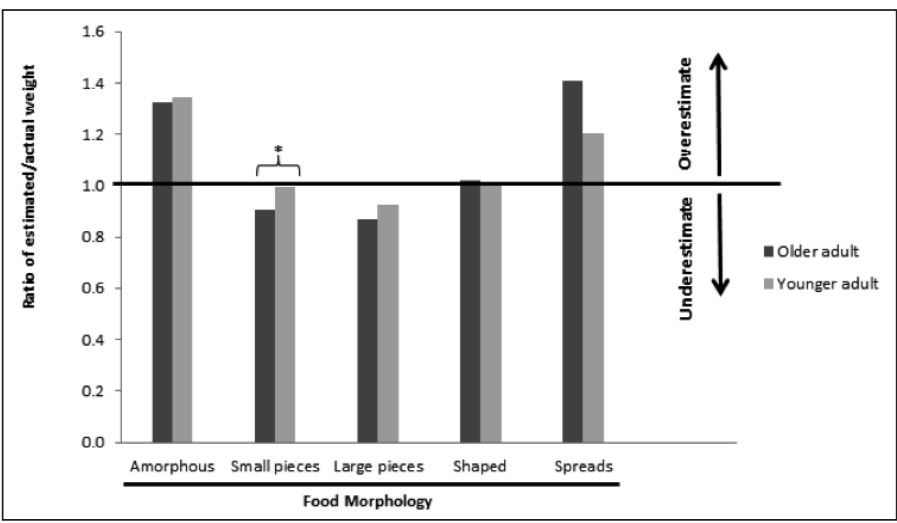

Results were obtained using univariate analysis of covariance, considering $* \mathrm{P}<0.05$ to be significantly different. A value greater than 1 indicates over reporting and a value less than 1 underreporting.

\section{Food photographic atlas versus food photographic software}

The ratio of estimated portion size weights to actual portion size weights assessed by participants using both traditional and computerised portion size assessment aids was also examined (Figure 2). There was no significant difference in the ability of the participants using the atlas versus the computer food photographs format when it came to the estimation of large pieces and spreads. There were significant differences between the traditional food atlas versus the computerised food atlas in the estimation of portion size of small pieces $(\mathrm{F}=5.51$, $\mathrm{df}=1, \mathrm{P}=0.019)$. The results show that using the traditional atlas method resulted in underestimation of small pieces, compared to the computerised version which yielded a more accurate estimation (i.e. a ratio closer to 1.0). Overestimation of amorphous food types and spreads was evident in the estimations using both portion size assessment aids whereas large pieces were underestimated using both assessment aids.

Older and younger adults versus nutritionists' ability to accurately estimate portion size

The mean ratio of estimated to actual assessment of portion size by nutritionists, older and younger adults is displayed in Table 3. A value greater than 1 denotes overestimation and a value less than 1 denotes underestimation. This is not a direct comparison as the nutritionists assessed the portion size of foods from photographs of meals whereas the older and younger adults assessed the portion size of self-served portions which were present at the time of estimation. On average, older adults tended to slightly overestimate compared to the other two groups although this was not statistically significant $(\mathrm{F}=0.971, \mathrm{df}=2, \mathrm{P}=0.379)$. Overall, the 3 test groups appear to be quite accurate in their estimation of portion size using both traditional and computerised assessment aids. The range of ratios highlights that there is more variability in the accuracy of the older and younger adults' estimates of portion size compared to the nutritionists.
Figure 2

The ability of participants to estimate portion size of different food morphology using food photographs presented in a traditional food atlas format or on a computer

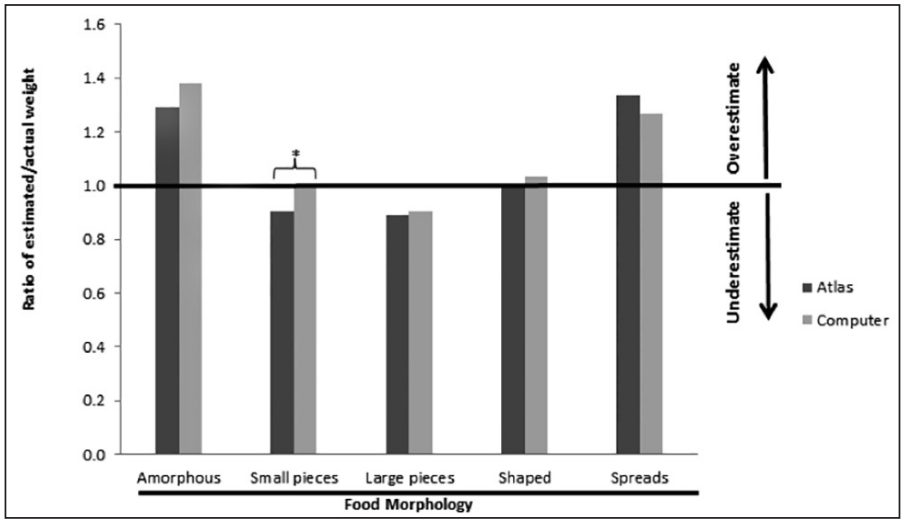

Results were obtained using univariate analysis of covariance, considering $* \mathrm{P}<0.05$ to be significantly different. A value greater than 1 indicates over reporting and a value less than 1 underreporting.

\section{Discussion}

Food photographic atlas versus food photographic software

Our findings suggest the estimation of portion sizes made using computerised portion size assessment tools provide similar estimates compared to traditional assessment aids. It is important to note that the older adult population in this study were highly motivated individuals and this level of motivation and high percentage of computer literate volunteers may not be representative of the general older adult population. The computer-based method displayed only two photographs at a time which participants anecdotally reported to prefer rather than seeing all 8 portion size images at one time (as displayed in the traditional food atlas).

Overall the vast majority of participants from all 3 study groups in this present study were relatively familiar with technology. Although the older adult participants in this study were highly motivated, the reported internet usage amongst these participants (72\% reported using the internet regularly) is comparable with the findings of an investigation into internet usage in the UK which found that $74.1 \%$ of adults aged 65 years and over used the internet on a regular basis (21). This suggests (although this was not investigated) that computerised portion size assessments aids may be acceptable to the general older adult population. In other studies researchers found that computer/ web based portion size images were acceptable to both children (22) and adults (11). In other groups such as those with low literacy or numeracy skills, computer/ web based portion size images were not always favourable and in some instances hand gestures such as an extended palm to indicate portion size of food consumed were preferred as opposed to web based images (23).

\section{The effect of age on portion size assessment}

There is a body of literature that suggests that younger adults 
THE JOURNAL OF NUTRITION, HEALTH \& AGINGC

Table 3

The ratio of estimated to actual portion size

\begin{tabular}{lccccc}
\hline & & & & & \multicolumn{2}{c}{$\begin{array}{c}\text { Range of estimates } \\
\text { Participant type }\end{array}$} & $\mathbf{n}$ & No. of estimates & Method & Mean ratio of estimated vs. actual & Minimum ratio & Maximum ratio \\
\hline Older adults & 40 & 598 & Direct estimation & 1.1 & 0.47 \\
Young adults & 41 & 629 & Direct estimation & 1.09 & 3.22 \\
Nutritionist & 25 & 374 & Estimation from photograph & 1.06 & 3.5 \\
\hline
\end{tabular}

Difference in the mean ratio of estimated vs. actual were investigated using univariate analysis of variance, there was no significant difference.

assess portion size more accurately than older adults $(5,6,8$, 11). In a study whereby the use of photographs to estimate food portion size was investigated, it was reported that being over the age of 65 was associated with small but statistically significant inaccuracies of portion size estimation when compared to other age populations (7). In a subsequent study it was also suggested that older adults have a tendency (only statistically significant for older adult males) to overestimate food portion size (5). In the current study whilst we did find that older adults had a tendency to overestimate spreads compared to younger adults this was not significantly different. In other studies, participants were asked to assess portion size from memory (5) (participants were asked to assess the portion size of food items 5 minutes after the consumption) which was not investigated in this present study which may in part explain the differences observed between the investigations. In a separate study investigating the ability of older adults to assess portion size, it was reported that there was no consistency in the direction of error (over or under estimation) across food groups (24).

The results show that older adults significantly underestimated small pieces when compared to younger adults. Although individual food types were not reported explicitly in this study, participants most commonly underestimated potatoes and cheese whereas curry, stew and spreads were most commonly overestimated. These foods are representative of the amorphous and pieces categories of food morphology. There is substantial evidence $(4,24,25)$ that amorphous foods and those foods eaten in smaller portions are less accurately reported. The literature suggests that the difficulty in estimating the portion sizes of small pieces such as vegetables is not limited to older adult populations. In a study whereby the ability of young adults to accurately estimate the portion sizes of small bite-sized vegetables was investigated, the results showed significant differences between the adult participants' estimation vs actual weight of vegetables (27).

Our findings suggest that both younger and older adults had similar difficulty accurately assessing food portion sizes.

The accuracy of dietary assessment by nutritionists, younger and older adults

The results from this study are in agreement with a body of literature that reports that portion sizes estimated by a layperson are often inaccurate. The main purpose of this study was to identify who is most accurate at assessing portion size for the development of the NANA system. Our findings suggest that all three groups have difficulties in accurately assessing portion size. Whilst these results are important for the development of improved dietary assessment tools, it is also important to consider the implications these findings may have in terms of how participants perceive portion sizes related food group based dietary guidelines.

The accuracy of portion size estimation from digital photographs has been compared with direct visual estimation (estimation of a portion size present in front of an individual) compared to the known weights of both (28). Portion size was assessed by trained researchers and the results highlighted both direct estimation and the estimation from photographs was highly correlated with the actual weights for each scenario (28). A limitation of our study was that nutritionists did not complete the direct visual portion size estimation as completed by the older and younger adults. However the study design tried to emulate that of a real life dietary assessment setting whereby a nutritionist would never directly observe the portion size consumed by individuals.

Although the nutritionists' ability to estimate individual food morphologies was not reported in this study (as these estimates were derived from photographs as opposed to direct observation which was the case for older and younger adults), the ranges of estimates show there is less variability in the estimates of portion sizes when assessed by a nutritionist. This suggests that nutritionists may assess portion size information more consistently than young and older adults. However the nutritionists provided inaccurate estimates for the portion sizes of large pieces particularly slices of tomatoes and cucumber. This suggests there may be difficulty in estimating these types of foods from photographs of meals. Similar difficulties were noted when the ability of nutrition students to assess the portion size of fruit and vegetables amongst other food items was investigated (29). The use of short training programs with portion size assessment aids prior to estimation has been shown to improve overall portion size estimation (25). However it is not evident in the literature whether a training exercise with portion size assessment aids would be beneficial to older adults.

\section{Conclusion}

Presently, the use of portion size images in dietary 


\section{PORTION ESTIMATION BY OLDER AND YOUNG ADULTS}

assessment pose minimal burden on participants compared to the alternative of weighed intakes. However, the findings of this study clearly highlight that using food portion size images to aid portion size assessment is associated with inaccuracies in both younger and older adults. Based on our results, we are unable to conclude that one age group is more accurate than the other. However, the computerised method was well received by younger and older adults alike and often preferred in comparison to traditional portion size assessment aids. It is important to consider these findings in the context of dietary assessment and the implications that portion size estimation by participants have on the accuracy of dietary records that require participants to estimate portion size.

This study also suggests that nutritionists may provide more accurate estimates of portion size with less variance in the estimates by nutritionists compared to younger and older adults. In terms of the development of the NANA system, it was concluded that higher accuracy would be achieved by a nutritionist making portion size assessment from a photograph of a meal and this was incorporated into the design of the system $(17,18)$. These results support the recent developments in dietary assessment and technology which have shown that taking the onus of portion size estimation off the individual and putting it on a trained researcher yields accurate results compared to weighed methods of dietary assessment $(13,14)$. However, many of the technological advancements in dietary assessment and portion size estimation have been developed for children and younger adults. This study suggests that the use of food photographs and the development of novel approaches could aid the accuracy of dietary assessment in all age groups.

Acknowledgments: The authors would like to thank Dr Alan Godfrey for his involvement in the development of the computerised portion size atlas and all the participants who were involved in this research. The authors gratefully acknowledge the use of the National Institute for Health Research, Sheffield Clinical Research Facility.

Financial Support: This study was funded by the Economic \& Social Research Council (ESRC) (RES-354-25-0003).

\section{Conflict of interest: The authors declare no conflict of interest.}

Ethical Standards Disclosure: This study was conducted according to the guidelines laid down in the Declaration of Helsinki and all procedures involving human subjects were approved by The University of Sheffield's Ethics Committee (SMBRER140). Written informed consent was obtained from all subjects.

Disclaimer: This is a summary of independent research funded by the ESRC and carried out at the NIHR Sheffield Clinical Research Facility. The views expressed are those of the author(s) and not necessarily those of the ESRC, NHS, the NIHR or the Department of Health.

Open Access: This article is distributed under the terms of the Creative Commons Attribution 4.0 International License (http://creativecommons.org/licenses/by/4.0/), which permits use, duplication, adaptation, distribution and reproduction in any medium or format, as long as you give appropriate credit to the original author(s) and the source, provide a link to the Creative Commons license and indicate if changes were made.

\section{References}

1. Nelson M and Bingham SA. Assessment of food consumption and nutrient intake. In: Design Concepts in Nutritional Epidemiology (Margetts, B. M. \& Nelson, M., eds.), 1997;pp 123-169, Oxford University Press, Oxford, UK.

2. Johansson G. Dietary assessments Use, design concepts, biological markers, pitfalls and validation. Halmstad University Press; 2014;20 p.
3. Gibson R. Principles of Nutritional Assessment. 2nd Edition. Oxford University Press, Oxford, 2005.

4. Howat PM, Mohan R, Champagne C, et al. Validity and reliability of reported dietary intake data. J Am Diet Assoc 1994;94(2):169-73. doi: 10.1016/0002-8223(94)90242-9

5. Nelson M, Atkinson M, Darbyshire S. Food photography II: use of food photographs for estimating portion size and the nutrient content of meals. Br J Nutr 1996;76(1):3149. doi: 10.1079/BJN19960007

6. Frobisher C, Maxwell SM. The estimation of food portion sizes: a comparison between using descriptions of portion sizes and a photographic food atlas by children and adults. J Hum Nutr Diet 2003;16(3):181-8. doi: 10.1046/j.1365-277X.2003.00434.x

7. Nelson M, Atkinson M, Darbyshire S. Food photography. I: The perception of food portion size from photographs. Br J Nutr 1994;72(5)649-63. doi: 10.1079/ BJN19940069

8. Young LR, Nestle MS. Portion sizes in dietary assessment: issues and policy implications. Nutr Rev 1995;53(6):149-58. doi: 10.1111/j.1753-4887.1995.tb01542.x

9. Hartley A. In the Craik and Salthouse Edition of The Handbook of Ageing and Cognition. Erlbaum, Hillsdale N, editor.

10. Foster E, Adamson AJ, Anderson AS, et al. Estimation of portion size in children's dietary assessment: lessons learnt. Eur J Clin Nutr 2009;63 Suppl 1:S45-9. doi: 10.1038/ejen.2008.64

11. Subar AF, Crafts J, Zimmerman TP, et al. Assessment of the accuracy of portion size reports using computer-based food photographs aids in the development of an automated self-administered 24-hour recall. J Am Diet Assoc 2010;110(1):55-64 doi:10.1016/j.jada.2009.10.007

12. Boushey CJ, Kerr DA, Wright J, et al. Use of technology in children's dietary assessment. Eur J Clin Nutr, 2009; 63 Suppl 1:S50-7. doi: 10.1038/ejen.2008.65

13. Jia W, Chen H-C, Yue Y, et al. Accuracy of food portion size estimation from digital pictures acquired by a chest-worn camera. Public Health Nutr 2014;17(8):1671-81. doi $10.1017 / \mathrm{s} 1368980013003236$

14. Lee CD, Chae J, Schap TE, et al. Comparison of known food weights with imagebased portion-size automated estimation and adolescents' self-reported portion size. J Diabetes Sci Technol, 2014;6(2):428-34. doi: 10.1177/193229681200600231

15. Wang DH, Kogashiwa M, Ohta S, et al. Validity and reliability of a dietary assessment method: the application of a digital camera with a mobile phone card attachment. J Nutr Sci Vitaminol 2002;48(6)498-504. doi: 10.3177/jnsv.48.498

16. Rollo ME, Ash S, Lyons-Wall P, et al. Trial of a mobile phone method for recording dietary intake in adults with type 2 diabetes: evaluation and implications for future applications. J Telemed Telecare 2011;17(6)318-23. doi: 10.1258/jtt.2011.100906

17. Astell AJ, Hwang F, Brown LJE, et al. Validation of the NANA (Novel Assessment of Nutrition and Ageing) touch screen system for use at home by older adults. Exp Gerontol 2014;60:100-7. doi: 10.1016/j.exger.2014.10.008

18. Timon CM, Astell AJ, Hwang F, et al. The validation of a computer-based food record for older adults: the Novel Assessment of Nutrition and Ageing (NANA) method. Br J Nutr 2015;113(4):654-64. doi: 10.1017/s0007114514003808

19. Nelson M, Atkinson M, Meyer J. A Photographic Atlas of Food Portion Sizes. MAFF Publications, London, 1997.

20. Gregory J, Foster K, Tyler H, Wiseman H. The Dietary and Nutritional Survey of British Adults. HMSO, London, 1990.

21. Office for National Statistics. Internet users in the UK. Available from:https:// www.ons.gov.uk/businessindustryandtrade/itandinternetindustry/bulletins/ internetusers/2016\#recent-internet-use-is-on-the-increase-for-those-aged-65-and-over. Accessed 28th of July 2016.

22. Foster E, Hawkins A, Simpson E et al. Developing an interactive portion size assessment system (IPSAS) for use with children. J Hum Nutr Diet 2014;27 Suppl 1:18-25. doi: 10.1111/jhn.12127

23. Chaudry B, Connelly K, Siek KA, et al. The Design of a Mobile Portion Size Estimation Interface for a Low Literacy Population. Int Conf Pervasive Comput Technol Healthc 2011;160-7. doi: 10.4108/icst.pervasivehealth.2011.246113

24. Ervin RB, Smiciklas-Wright H. Accuracy in estimating and recalling portion sizes of foods among elderly adults. Nutr Res 2001;21(5):703-13. doi: 10.1016/s0271 5317(01)00288-3

25. Bryd-Bredbenner C, Schwartz J. (The effect of practical portion size measurement aids on the accuracy of portion size estimates made by young adults. J Hum Nutr Diet 2004;17(4). doi: 10.1111/j.1365-277x.2004.00534.x

26. Venter CS, MacIntyre UE, Vorster HH. The development and testing of a food portion photograph book for use in an African population. J Hum Nutr Diet 2000;13(3):205-18 doi: 10.1046/j.1365-277x.2000.00228.x

27. Nørnberg TR, Houlby L, Jørgensen LN et al. Do we know how much we put on the plate? Assessment of the accuracy of self-estimated versus weighed vegetables and whole grain portions using an Intelligent Buffet at the FoodScape Lab. Appetite 2014;81:162-7 doi: 10.1016/j.appet.2014.06.007

28. Williamson DA, Allen HR, Martin PD, et al. Comparison of digital photography to weighed and visual estimation of portion sizes. J Am Diet Assoc, 2003;103(9):1139-45. doi: 10.1016/s0002-8223(03)00974-x

29. Japur CC, Diez-Garcia RW. Food energy content influences food portion size estimation by nutrition students. J Hum Nutr Diet 2010;23(3):272-6. doi: 10.1111/j.1365-277x.2010.01042.x 